\section{Dr. Henderson, et al reply}

\section{To the Editor:}

We are grateful for the thoughtful commentary of Dr. Eberlein ${ }^{1}$ on our recent study of shrinking lung syndrome (SLS) ${ }^{2}$.

We reported that SLS was associated with 2 abnormalities of pulmonary physiology: (1) reduced inspiratory force at high but not low lung volumes, a pattern noted in pleuritis; and (2) markedly reduced lung compliance, in the absence of interstitial lung disease as determined by chest tomography and gas diffusion. Recognizing that SLS is frequently associated with episodes of chest pain and/or small pleural effusions, and presents clinically as gradually progressive dyspnea that stops short of respiratory failure, we proposed a new working model for SLS. Briefly, we hypothesized that SLS begins with pleuritis that triggers voluntary and reflex splinting of the chest wall. Chronic hypoinflation leads to gradual but persistent impairment of compliance, a process recognized in patients with high spinal cord injuries and in other conditions associated with chronically low lung volumes and thought to be mediated by remodeling of the pulmonary parenchyma. Reduced lung compliance in turn favors worsening hypoinflation, setting up a cycle of gradually progressive pulmonary restriction that stabilizes once further hypoinflation is prohibited by the patient's respiratory drive.

Dr. Eberlein points out that remodeling is not the only pathway to hypoinflation-induced impairment of lung compliance. As he notes, studies in healthy volunteers show that chest strapping or voluntary low-volume breathing also produces a rapid loss of lung compliance, one that is not completely understood but typically attributed to an increase in alveolar surface tension. Could this effect be the mechanism of compliance loss in SLS, without invoking structural changes in the lung, for which, as Dr. Eberlein notes, there is little understanding at the histopathological level in either SLS or other chronically volume-restricted states?

We agree that this is a key question and recognize that multiple factors could contribute to loss of compliance in individuals with SLS, potentially coming into play at different phases of the disease. Indeed, improvement with treatment in many patients points to a reversible component in SLS-associated lung dysfunction. However, we suggest that the physiology described is unlikely to be the major determinant of the SLS phenotype. As Dr. Eberlein notes, impairment of compliance by chest strapping is quickly reversed $-\mathrm{a}$ few deep breaths are sufficient to restore normal compliance measures ${ }^{3,4}$. This was not the case with our patients with SLS, in whom compliance remained low through the course of pulmonary testing that included maximal respiratory efforts. Further, in 1 patient studied months after symptomatic improvement, we found that lung compliance remained markedly low. These findings suggest that the physiology of SLS more closely resembles that of spinal cord injury, where chronic hypoventilation is associated with a fixed impairment of compliance that cannot be reversed even after mechanical ventilation to maximally inflate the lung ${ }^{5,6}$. Careful investigation of these patients has suggested that their pulmonary physiology is best represented by a model of alveolar loss, with normal elastic/surface tension measurements of the residual alveoli ${ }^{7,8}$. Therefore, we have proposed that the same kind of parenchymal changes ("remodeling") occur in SLS, potentially accounting for the progressive and potentially persistent character of the restrictive changes.

Dr. Eberlein raises the interesting possibility that an abnormality in surfactant, as a major determinant of alveolar surface tension, may contribute to SLS. This hypothesis was suggested in the original description of $\mathrm{SLS}^{9}$, but to our knowledge no supporting data have since emerged, although the question has not yet been thoroughly studied. Surfactant represents a complex lipoprotein mixture. Neonates with surfactant deficiency present with widespread atelectasis, and atelectasis and recurrent infections are also noted in children and adults with mutations affecting surfactant genes. These phenotypes are not particularly reminiscent of SLS. Further, SLS is commonly preceded by clinical or radiological evidence for pleuritis, an association that would not be expected if the primary process were a surfactant abnormality. Thus, while surfactant may play some role in SLS physiology, we doubt that surfactant abnormalities represent a primary factor in disease pathogenesis.

These considerations notwithstanding, we are indebted to Dr. Eberlein for highlighting the possible role of rapid and reversible as well as slowly progressive and fixed causes of impaired lung compliance in SLS. His comments serve as a reminder that the model we have proposed is a working hypothesis whose validation will require further study, including longterm followup of additional patients and examination of affected lung tissue to define the histopathological basis of persistently impaired lung compliance.

LAUREN A. HENDERSON, MD, Division of Immunology, Boston Children's Hospital, the Department of Anesthesia and Critical Care, Beth Israel Deaconess Medical Center; STEPHEN H. LORING, MD,

Department of Anesthesia and Critical Care, Beth Israel Deaconess Medical Center; PAUL F. DELLARIPA, MD, Division of Rheumatology, Immunology, and Allergy, Brigham and Women's Hospital; and PETER A. NIGROVIC MD, Division of Rheumatology, Immunology, and Allergy, Brigham and Women's Hospital, and Division of Immunology, Boston Children's Hospital, Boston, Massachusetts, USA. Address correspondence to Dr. P.A. Nigrovic, Division of Rheumatology, Immunology and Allergy, Brigham and Women's Hospital, Smith Building Room 516b, One Jimmy Fund Way, Boston, MA 02115, USA.

E-mail: pnigrovic@partners.org

\section{REFERENCES}

1. Eberlein M. Shrinking lung syndrome as a manifestation of pleuritis: are surface forces and surfactant the pathophysiological link? J Rheumatol 2013;40:1926.

2. Henderson LA, Loring SH, Gill RR, Liao KP, Ishizawar R, Kim S, et al. Shrinking lung syndrome as a manifestation of pleuritis: a new model based on pulmonary physiological studies. J Rheumatol 2013;40:273-81.

3. Butler J, Caro CG, Alcala R, Dubois AB. Physiological factors affecting airway resistance in normal subjects and in patients with obstructive respiratory disease. J Clin Invest 1960;39:584-91.

4. Estenne M, Gevenois PA, Kinnear W, Soudon P, Heilporn A, De Troyer A. Lung volume restriction in patients with chronic respiratory muscle weakness: the role of microatelectasis. Thorax 1993;48:698-701.

5. De Troyer A, Deisser P. The effects of intermittent positive pressure breathing on patients with respiratory muscle weakness. Am Rev Respir Dis 1981;124:132-7.

6. McCool FD, Mayewski RF, Shayne DS, Gibson CJ, Griggs RC, Hyde RW. Intermittent positive pressure breathing in patients with respiratory muscle weakness. Alterations in total respiratory system compliance. Chest 1986;90:546-52.

7. De Troyer A, Heilporn A. Respiratory mechanics in quadriplegia. The respiratory function of the intercostal muscles. Am Rev Respir Dis 1980;122:591-600.

8. De Troyer A, Borenstein S, Cordier R. Analysis of lung volume restriction in patients with respiratory muscle weakness. Thorax 1980;35:603-10.

9. Hoffbrand BI, Beck ER. "Unexplained" dyspnoea and shrinking lungs in systemic lupus erythematosus. Br Med J 1965;1:1273-7.

J Rheumatol 2013;40:11; doi:10.3899/jrheum.130897 